\title{
ANALYTIC APPROXIMATIONS TO GALAXY CLUSTERING
}

\author{
H.J. MO \\ Max-Planck-Institut für Astrophysik \\ Karl-Schwarzschild-Str. 1, 85748 Garching, Germany
}

\begin{abstract}
We discuss some recent progress in constructing analytic approximations to the galaxy clustering. We show that successful models can be constructed for the clustering of both dark matter and dark matter haloes. Our understanding of galaxy clustering and galaxy biasing can be greatly enhanced by these models.
\end{abstract}

\section{Introduction}

The large scale structure of the Universe is believed to have developed from small perturbations (usually assumed to be Gaussian) of the matter density field by gravitational instabilities. Under these assumptions the clustering pattern and velocity field observed today are determined by the initial conditions via the perturbation power spectrum $[P(k)]$ and the cosmological parameters such as $\Omega_{0}$, the cosmic density parameter. It is therefore possible to derive constraints on model parameters from the observed density and velocity distributions of galaxies.

There are two fundamental problems to be addressed: First, since the galaxy distribution may be biased relative to the mass density field, we need to understand such bias before making meaningful comparisons between models and observations. Second, even if the observed galaxy distribution traces the matter distribution, we still need to understand how the observed distribution is related to a cosmogonical model. This is by no means trivial, because the clustering pattern and velocity field observed today are nonlinear. N-body simulations are usually invoked to find a solution to these problems. However, such simulations are limited both in resolution and in dynamical range, and can be difficult to interpret. Our understanding of the underlying physics can be greatly enhanced by simple physical models and the analytic approximations they provide. 
In this article we summarize some of our recent progress in connection to the problems mentioned above. We show that successful semianalytic models can be constructed for the clustering of both dark matter and dark matter haloes. Such models can enhance significantly our understanding of both the nonlinear evolution of galaxy clustering and galaxy biasing.

\section{Cosmogonies}

The models present below are for CDM-like cosmogonies. The cosmology is described by the cosmological matter density $\left(\Omega_{0}\right)$, the cosmological constant $\left(\lambda_{0}\right)$ and the Hubble constant $\left(H_{0}=100 h \mathrm{~km} \mathrm{~s}^{-1} \mathrm{Mpc}^{-1}\right)$. The initial power spectrum is

$$
\begin{gathered}
P(k) \propto k T^{2}(k), \\
T(k)=\frac{\ln (1+2.34 q)}{2.34 q}\left[1+3.89 q+(16.1 q)^{2}+(5.46 q)^{3}+(6.71 q)^{4}\right]^{-1 / 4}
\end{gathered}
$$

where $q \equiv k /\left(\Gamma h \mathrm{Mpc}^{-1}\right), \Gamma \equiv \Omega_{0} h$ (Bardeen et al. 1986). The RMS mass fluctuation in top-hat windows with radius $R, \sigma(R)$, is defined by

$$
\sigma^{2}(R)=\int_{0}^{\infty} \frac{d k}{k} \Delta^{2}(k) W^{2}(k R)
$$

where $W(x)$ is the Fourier transform of the top-hat window function, and

$$
\Delta^{2}(k)=\left(1 / 2 \pi^{2}\right) k^{3} P(k)
$$

is the power variance. We normalize $P(k)$ by specifying $\sigma_{8} \equiv \sigma\left(8 h^{-1} \mathrm{Mpc}\right)$.

\section{Low-Order Statistics of Dark Matter Distribution}

\subsection{MASS CORRELATION FUNCTION}

The evolved two-point correlation function $\xi(r)$ is related to the evolved power variance $\Delta_{E}^{2}(k)$ by

$$
\xi(r)=\int_{0}^{\infty} \frac{d k}{k} \Delta_{E}^{2}(k) \frac{\sin k r}{k r} .
$$

Thus, in order to get $\xi(r)$ we need an expression for $\Delta_{E}(k)$. Following the original argument of Hamilton et al. (1991), Jain, Mo \& White (1995), Padmanabhan et al. (1996) and Peacock \& Dodds (1996, hereafter PD) have obtained fitting formulae which relate $\Delta_{E}$ to $P(k)$ for a given cosmological model. The latest version of such a fitting formula is given in PD. The solid curve in Fig.1 shows the prediction of such a formula for $\xi(r)$ in the 


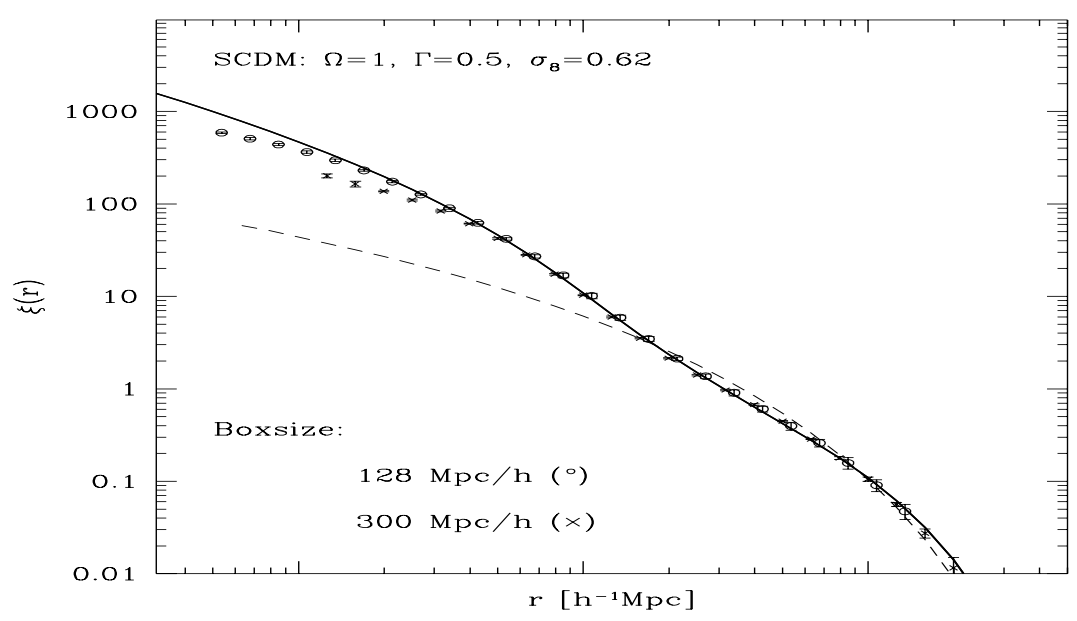

Figure 1. Predicted two-point correlation function of mass (solid curve) compared with simulation results (symbols). The dashed curve is given by the linear power spectrum.
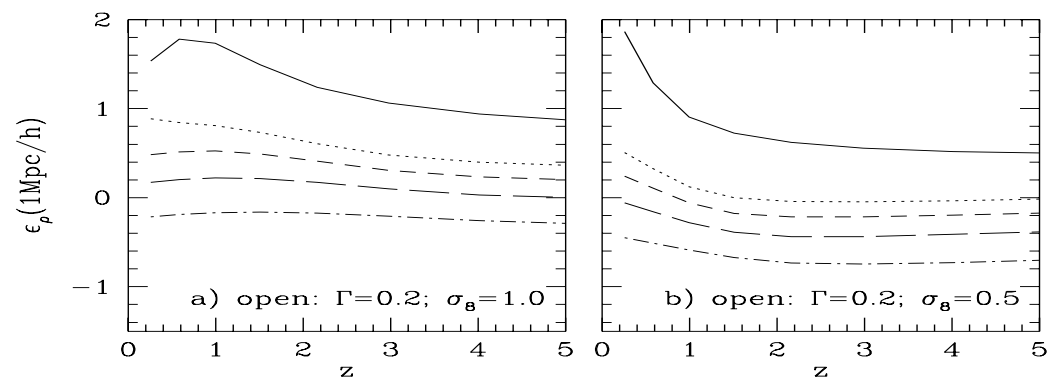

Figure 2. The redshift-evolution parameter of the mass correlation function as a function of redshift, for open cosmologies with $\Omega_{0}=0.1,0.2,0.3,0.5$ and 1 (from bottom up).

standard cold dark matter (SCDM) model with $\Omega_{0}=1, \lambda_{0}=0, h=0.5$ and $\sigma_{8}=0.62$ (see Mo, Jing \& Börner 1997, hereafter MJB, for details). Comparing it with the N-body results, we see clearly that the fitting formula works well. This is true for various other cosmogonic models studied.

\subsection{REDSHIFT EVOLUTION OF MASS CORRELATION FUNCTION}

The redshift evolution of the two-point correlation function is usually parametrized by the form

$$
\xi(r, z)=\xi(r, 0)(1+z)^{-\left(3+\epsilon_{\rho}\right)},
$$

where $\xi(r, z)$ is the amplitude of the two-point correlation function at phys$i c a l$ radius $r$ at redshift $z, \epsilon_{\rho}$ is a parameter to describe the time evolution. If $\xi(r) \propto r^{-\gamma}$, then $\epsilon_{\rho}=\gamma-1$ for the linear growth in an Einstein-de Sitter 


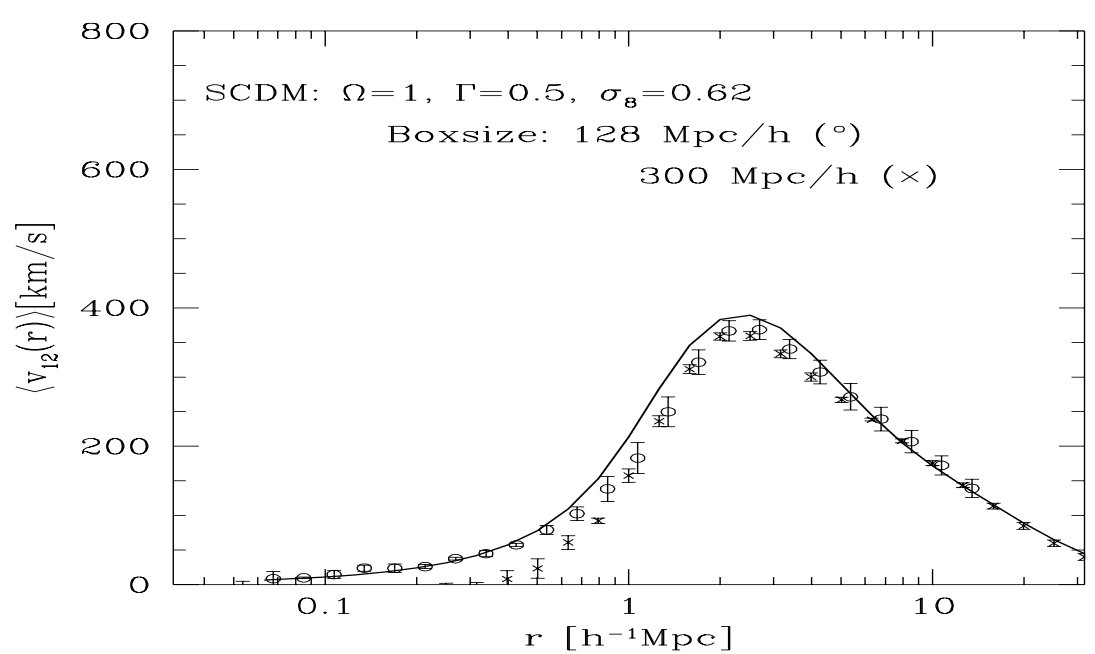

Figure 3. Mean pairwise peculiar velocity of dark matter particles. Curve is the model prediction; symbols are the simulation results.

universe, $\epsilon_{\rho}=3-\gamma$ for clustering patterns fixed in comoving space, and $\epsilon_{\rho}=0$ for stable clustering (i.e. clustering patterns fixed in proper coordinates). Given the model in $\S 3.1$, it is straightforward to obtain $\epsilon_{\rho}$. Fig. 2 shows $\epsilon_{\rho}$ as a function of $z$ for open cosmologies with various $\Omega_{0}$. The value of $\epsilon_{\rho}$ at $z$ is obtained by fitting $\xi\left(r=1 h^{-1} \mathrm{Mpc}, z^{\prime}\right)$ in the redshift interval, $z^{\prime}=0 \rightarrow z$. The evolution is more rapid (i.e. $\epsilon_{\rho}$ larger) for universes with larger $\Omega_{0}$, since linear structures grow faster in a high- $\Omega_{0}$ universe. For high $\Omega_{0}$, the evolution is faster at $z \sim 1$, because of nonlinear evolution. At late time when clustering on $r \sim 1 h^{-1} \mathrm{Mpc}$ becomes stable, the evolution becomes slower.

\subsection{MEAN PAIRWISE PECULIAR VELOCITIES}

From the pair conservation equation (Peebles 1980, §71), the ensemble (pair weighted) average of the pairwise peculiar velocity $\left\langle v_{12}(r)\right\rangle \equiv\langle[\mathbf{v}(\mathbf{x})-\mathbf{v}(\mathbf{x}+$ $\mathbf{r})] \cdot \hat{\mathbf{r}}\rangle$ can be written as

$$
\frac{\left\langle v_{12}(r)\right\rangle}{H(a) r}=-\frac{1}{3} \frac{1}{[1+\xi(y, a)]} \frac{\partial \bar{\xi}(y, a)}{\partial \ln a}
$$

where $r$ is the proper, and $y$ the comoving, separation between the pairs; $H$ is the Hubble's constant at expansion factor $a ; \bar{\xi}(y, a) \equiv\left(3 / y^{3}\right) \int_{0}^{y} y^{2} d y \xi(y, a)$. Thus, to obtain $\left\langle v_{12}(r)\right\rangle$, we need to work out $\partial \Delta_{E}(k, a) / \partial a$. This can be done directly from the fitting formula of $\Delta_{E}(k, a)$ (see MJB). Fig. 3 shows the comparison between the model prediction and the simulation results 
for the SCDM model. The agreement between the two is remarkably good, and this is true for many other cosmogonic models studied.

\subsection{COSMIC ENERGY EQUATION}

The (density weighted) mean square peculiar velocity of mass particles $\left\langle v_{1}^{2}\right\rangle$ is related to the two-point correlation function by the cosmic energy equation:

$$
\frac{d}{d a} a^{2}\left\langle v_{1}^{2}\right\rangle=4 \pi G \bar{\rho} a^{3} \frac{\partial I_{2}(a)}{\partial \ln a},
$$

where $\bar{\rho}$ is the mean density of the universe, and

$$
I_{2}(a) \equiv \int_{0}^{\infty} y d y \xi(y, a)=\int_{0}^{\infty} \frac{d k}{k} \frac{\Delta_{E}^{2}(k, a)}{k^{2}}
$$

Integrating eqn. (8) once, we have

$$
\left\langle v_{1}^{2}\right\rangle=\frac{3}{2} \Omega(a) H^{2}(a) a^{2} I_{2}(a)\left[1-\frac{1}{a I_{2}(a)} \int_{0}^{a} I_{2}(a) d a\right] .
$$

In the linear case, $I_{2}(a) \propto D^{2}(a)$, where $D(a)=a g(a)[g(a)$ is the linear growth factor; $a_{0}=1$ ] and

$$
\left\langle v_{1}^{2}\right\rangle=\frac{3}{2} \Omega(a) H^{2}(a) a^{2} I_{2}(a)\left[1-\frac{1}{a D^{2}(a)} \int_{0}^{a} D^{2}(a) d a\right] .
$$

We found that eqn.(11) is a good approximation (to an error of $<10 \%$ ) to eqn.(10) for all realistic cases. Thus, for a given cosmogonic model, we can easily obtain $\left\langle v_{1}^{2}\right\rangle$. For various cosmogonies, the model predictions fit the simulation results to an accuracy better than 10 percent (see MJB).

\subsection{PAIRWISE PECULIAR VELOCITY DISPERSION}

The relative velocity dispersion of particle pairs of separation $r$ is defined as $\left\langle[\mathbf{v}(\mathbf{x})-\mathbf{v}(\mathbf{x}+\mathbf{r})]^{2}\right\rangle^{1 / 2}$. In Fig. 4 we show (by symbols) the dispersion of the pairwise peculiar velocities projected along the separations of particle pairs $\left[\left\langle v_{12}^{2}(r)\right\rangle^{1 / 2}\right]$ in the N-body simulations. The main features of $\left\langle v_{12}^{2}(r)\right\rangle^{1 / 2}$ are (a) monotonic rise at small $r$; (b) saturation at large $r$; (c) a maximum at medium $r$. As shown in MJB, these features can all be explained by physical arguments.

Based on the N-body results we make the following ansatz for $\left\langle v_{12}^{2}(r)\right\rangle$ :

$$
\left\langle v_{12}^{2}(r)\right\rangle^{1 / 2}=\Omega^{0.5} H r_{c} \phi\left(r / r_{c}\right),
$$




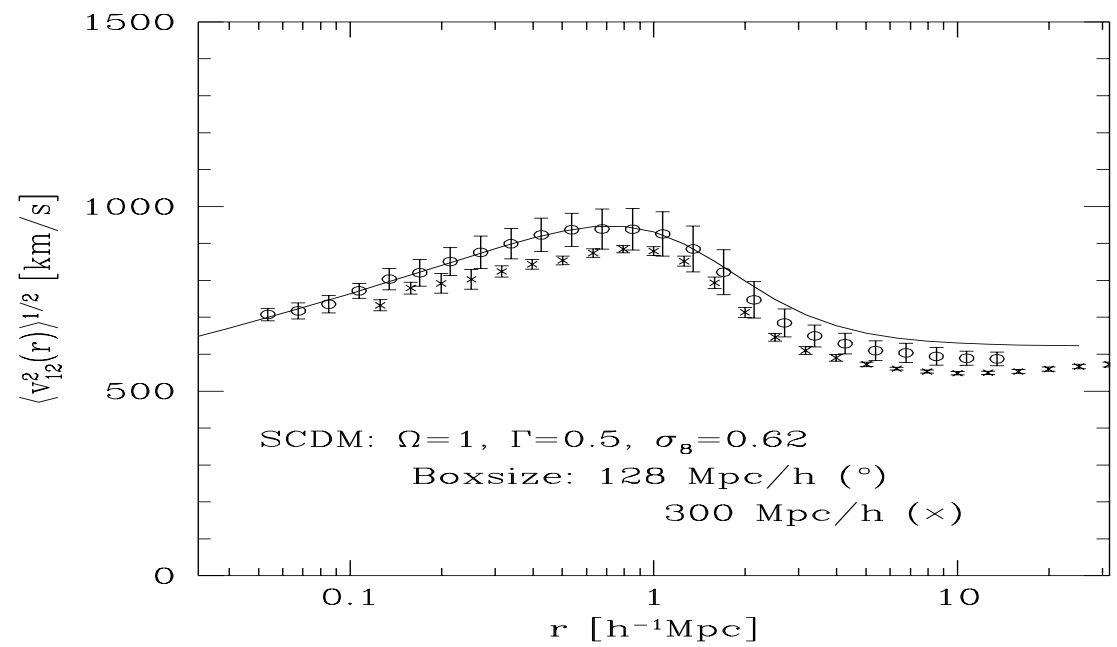

Figure 4. Pairwise peculiar velocity dispersion of dark matter particles. Curve is the model prediction; symbols are the simulation results.

where $\phi(x)$ is a universal function and $r_{c}$ is a nonlinear scale. We choose $r_{c}$ to be the virial radius of $M^{*}$ haloes, $r_{v}^{*}$. For a given power spectrum, the linear radius of $M^{*}$ haloes, $r_{0}^{*}$, is given by $\sigma\left(r_{0}^{*}\right)=1$. The relation between $r_{v}^{*}$ and $r_{0}^{*}$ in different cosmological models can be obtained analytically, as discussed in detail in MJB. We approximate the functional form of $\phi(x)$ by:

$$
\phi(x)=\frac{\phi_{\infty}\left(1+B x^{-\beta}\right)+A x^{-\alpha}}{1+V[g(a)]^{0.35}[\Omega(a)]^{0.2} x^{-(\alpha+\kappa)}},
$$

where $\phi_{\infty}=\sqrt{\frac{2}{3}}\left\langle v_{1}^{2}\right\rangle^{1 / 2} /\left(H r_{c} \Omega^{0.5}\right) ; A, B, V, \alpha>\beta>0$, and $\kappa>0$ are constant. $\left\langle v_{12}^{2}(r)\right\rangle$ is forced to have the large separation asymptotic value for uncorrelated pairs. For $r \rightarrow 0,\left\langle v_{12}^{2}(r)\right\rangle^{1 / 2} \propto x^{\kappa}$ so that it increases with $r$ as a power law. The solid curve in Fig. 4 shows the prediction of our fitting formula with

$$
\begin{gathered}
A=58.67 ; \quad B=-0.3770 ; \quad V=4.434 ; \\
\alpha=2.25 ; \quad \beta=1.90 ; \quad \kappa=0.15 .
\end{gathered}
$$

The fit to the simulation data is reasonably good.

\section{Spatial Clustering of Dark Matter Haloes}

So far we have discussed the clustering properties of dark matter. To compare model predictions with the observed galaxy distribution, we also need to understand how the galaxy distribution is related to the dark matter 
distribution. The bias of the galaxy distribution relative to the mass distribution can be obtained once we know how galaxies form in the mass density field. However, some progress can still be made before the details of galaxy formation is understood. In the standard scenario of galaxy formation, a gravitationally dominant dissipationless component of dark matter is assumed to aggregate into dark matter clumps (dark matter haloes), galaxies then form by the cooling and condensation of gas within these dark haloes (e.g. Kauffmann 1997). It is therefore important to approach the problem of galaxy biasing by first understanding how dark matter haloes are distributed relative to the mass. In the following we show that simple analytic models for such relations can be constructed (see Mo \& White 1996, MW).

\subsection{BIAS RELATION}

We define dark matter haloes as virialized clumps of dark matter. In the spherical collapse model, a dark matter halo is characterized by its mass, $M$, and the redshift, $z$, when it is assembled. To describe the relation between halo and mass distributions we define a bias relation,

$$
\delta_{h}(R)=b(R, \delta, M, z) \delta(R),
$$

where $\delta(R)=[\rho(R)-\bar{\rho}] / \bar{\rho}$ is the overdensity of matter in a sphere of radius $R, \delta_{h}(R)$ is the same overdensity for dark matter haloes. Suppose the conditional density of dark matter haloes within a sphere of radius $R$ is $n_{h}(M, z \mid \delta, R)$. (This is the number density of dark matter haloes within a sphere of radius $R$, given that the mean mass overdensity within this sphere is $\delta$.) The bias relation can then be written as

$$
\delta_{h}(M, z \mid \delta, R)=\frac{n_{h}(M, z \mid \delta, R)}{\bar{n}_{h}(M, z)}-1,
$$

where $\bar{n}_{h}(M, z)$ is the mean number density of haloes in the universe, given by the Press-Schechter formalism (Press \& Schechter 1974, PS):

$$
\bar{n}_{h}(M, z) d M=-\sqrt{\frac{2}{\pi}} \frac{\bar{\rho}}{M} \frac{\delta_{z}}{\sigma^{2}(r)} \frac{d \sigma}{d M} \exp \left[-\frac{\delta_{z}^{2}}{2 \sigma^{2}(r)}\right] d M,
$$

where $M=(4 \pi / 3) \bar{\rho} r^{3}, \delta_{z}=\delta_{c} D\left(a_{0}\right) / D(a), \delta_{c} \approx 1.686$. The conditional density, $n_{h}(M, z \mid \delta, R)$, can be obtained by an extension of the PS formalism (e.g. Bower 1991):

$$
\begin{aligned}
n_{h}\left(M, z \mid \delta_{0}, R_{0}\right) d M & =-\sqrt{\frac{2}{\pi}} \frac{\bar{\rho}}{M} \frac{\left(\delta_{z}-\delta_{0}\right) \sigma(r)}{\left[\sigma^{2}(r)-\sigma^{2}\left(R_{0}\right)\right]^{3 / 2}} \frac{d \sigma(r)}{d M} \\
& \times \exp \left\{-\frac{\left(\delta_{z}-\delta_{0}\right)^{2}}{2\left[\sigma^{2}(r)-\sigma^{2}\left(R_{0}\right)\right]}\right\} d M,
\end{aligned}
$$



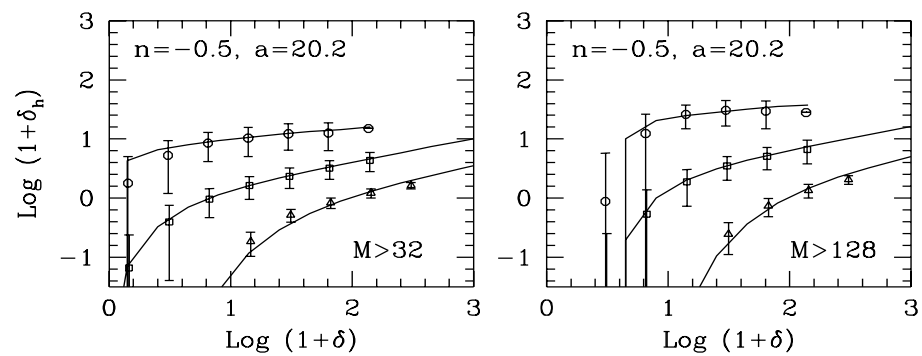

Figure 5. Bias relation $\delta_{h}(\delta)$ in a scale free model with $n=-0.5$. Results are shown for spheres with radii $R / L=0.02,0.05$ and 0.13 ( $L$ : the side of the simulation box). The results for $R / L=0.05$ and 0.13 are shifted by 1 and 2 decades along the horizontal axis.

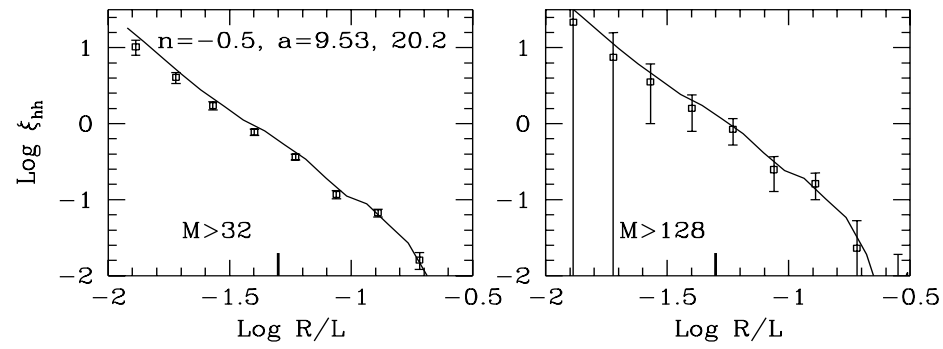

Figure 6. Predicted halo-halo two-point correlation functions (solid curves) compared to those from simulations (symbols).

where $\delta_{0}$ is the linear mass overdensity of spherical regions with Lagrangian radius $R_{0}$. Assuming spherical collapse, we have $R_{0}=[1+\delta(R)]^{1 / 3} R$, and $\delta_{0}$ is determined by $\delta(R)$ and $R$ through the spherical collapse model (see MW for details). With these the bias relation is fixed. Fig. 5 shows the bias relation for haloes in a scale free model with $P(k) \propto k^{-0.5}$. It is clear that our simple model works reasonably well.

\subsection{TWO-POINT CORRELATION FUNCTION OF HALOES}

When $\delta \ll 1$ and $r \ll R_{0}$, the bias relation can be written as

$$
\delta_{h}(R ; M, z)=b(M, z) \delta(R) ; \quad b(M, z)=1+\frac{1}{\delta_{z}}\left[\frac{\delta_{z}^{2}}{\sigma^{2}(r)}-1\right]
$$

Thus the bias factor $b$ depends only on $M$ and $z$. Under the assumption of linear bias,

$$
\xi_{h}(r)=b^{2}(M, z) \xi(r),
$$



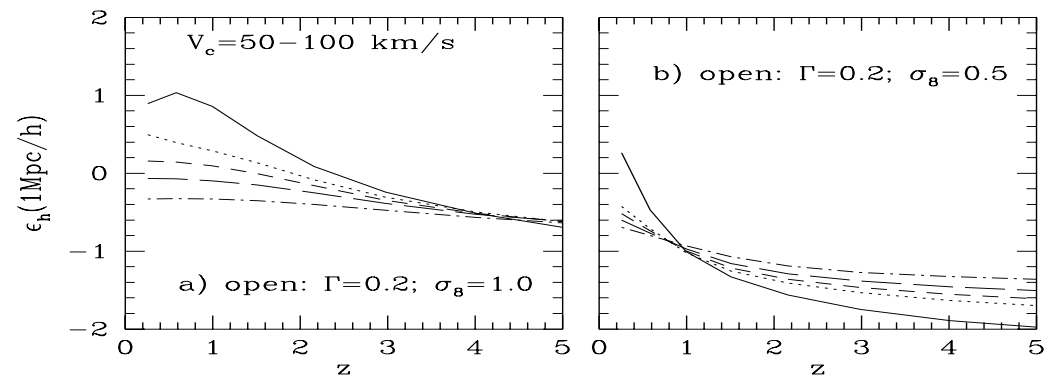

Figure 7. The redshift-evolution parameter of the halo-halo correlation function for haloes selected according to the first selection rule (see text). Results are shown for open cosmologies with $\Omega_{0}=0.1,0.2,0.3,0.5$ and 1 (from dot-dashed to solid curves).
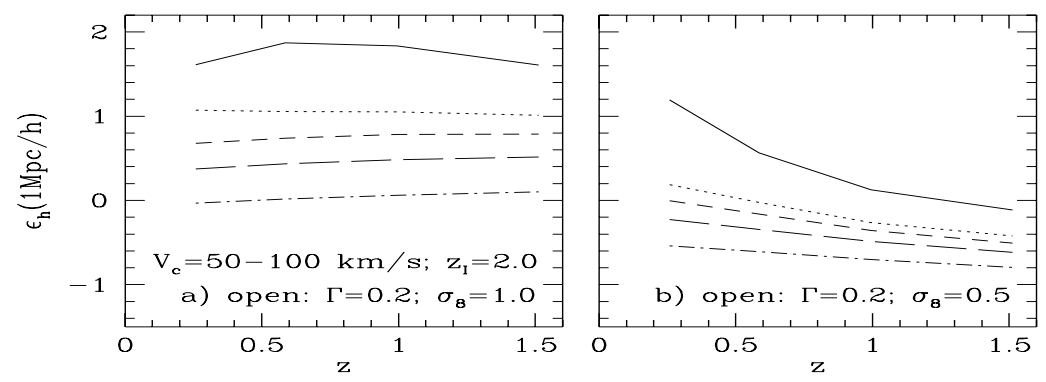

Figure 8. The same as Fig. 7 for haloes selected according to the second selection rule.

where $\xi(r)$ is the two-point correlation function of mass. Fig. 6 shows the two-point correlation functions for haloes in a scale free model with $P(k) \propto$ $k^{-0.5}$. The simple model works reasonably well.

\subsection{REDSHIFT EVOLUTION OF HALO CORRELATION FUNCTION}

As in $§ 3.2$, we parametrize the redshift evolution of $\xi_{h}$ by

$$
\xi_{h}(r, z)=\xi_{h}(r, 0)(1+z)^{-\left(3+\epsilon_{h}\right)} .
$$

Unlike the mass correlation function $\xi$, the evolution of $\xi_{h}$ depends on how dark matter haloes are selected. We consider two different selections. In the first, haloes are selected at the same time when the correlation function is calculated. This case is relevant for galaxies, if galaxies merge as fast as their dark haloes. In the second, haloes are selected at an earliear epoch than when their correlation function is calculated. This case is relevant for galaxies, if they remain distinct after their haloes merge. Fig. 7 shows $\epsilon_{h}$ as a function of $z$ in open cosmologies with two choices of $\sigma_{8}$. Here haloes are selected according to the first selection. As before, the value of $\epsilon_{h}$ at $z$ is obtained by fitting $\xi_{h}\left(r=1 h^{-1} \mathrm{Mpc}, z^{\prime}\right)$ in the redshift interval 
$z^{\prime}=0 \rightarrow z$. In all cases, the halo correlation function evolves less rapidly than the mass correlation function (see Fig. 2), because haloes with fixed circular velocities are more biased at higher redshifts. The evolution is also less rapid for a lower $\sigma_{8}$, because of the higher degree of bias involved. Fig. 8 shows the same results for haloes selected according to the second selection. In this case, the evolution of $\xi_{h}$ is faster than that of $\xi$, because the haloes (with $V_{c}=50-100 \mathrm{~km} \mathrm{~s}^{-1}$ ) are antibiased relative to the mass at the time of selection $(z=2)$. For massive haloes which are (positively) biased relative to the mass, the evolution of their correlation function is slower than that of the mass. The evolution is also slower for a lower $\sigma_{8}$, because of the increased degree of bias (or decreased degree of antibias). The results in this subsection suggest that one needs to be very cautious when interpreting the redshift evolution of galaxy correlation function. Without knowing in detail what population the observed galaxies are, it is difficult to infer the time evolution of the mass correlation from the correlation functions of these galaxies.

\section{Discussion}

The models presented above show how the low order statistics of the density and velocity distributions are determined by cosmogonies. Thus they can be used to construct statistical measures of the density and peculiar velocity fields to constrain cosmogonic models by observations. In particular, the models can help in the reconstruction of cosmogonic parameters from measurements in redshift space. There are also other applications. MJB discuss the dependence of the small scale pairwise peculiar velocity dispersion on the presence (or absence) of rich clusters of galaxies. Mo, Jing \& White (1996) use the model to study the correlation of galaxy clusters. Mo, Jing \& White (1997) extend the model to high-order correlations of dark matter haloes and density peaks.

\section{References}

1. Bardeen J., Bond J.R., Kaiser N., Szalay A.S., 1986, ApJ, 304, 15

2. Bower R.J., 1991, MNRAS, 248, 332

3. Hamilton A.J.S., Kumar P., Lu E., Matthews A., 1991, ApJ, 374, L1

4. Jain B., Mo H.J., White S.D.M., 1995, MNRAS, 276, L25

5. Kauffmann G., 1997, this volume

6. Mo H.J., Jing Y.P., Börner G., 1997, MNRAS, in press (MJB)

7. Mo H.J., Jing Y.P., White S.D.M., 1996, MNRAS, 282, 1096

8. Mo H.J., Jing Y.P., White S.D.M., 1997, MNRAS, 284, 189

9. Mo H.J., White S.D.M., 1996, MNRAS, 282, 347 (MW)

10. Padmanabhan T., Cen R., Ostriker J.P., Summers F.J., 1996, preprint

11. Peacock J.A., Dodds S.J., 1996, MNRAS, 280, L19 (PD)

12. Peebles P.J.E., 1980, The Large-Scale Structure of the Universe, Princeton

13. Press W.H. \& Schechter P., 1974, ApJ, 187, 425 (PS) 